This item was submitted to Loughborough's Research Repository by the author.

Items in Figshare are protected by copyright, with all rights reserved, unless otherwise indicated.

\title{
Experimental investigation of turbulent flame propagation and pressure oscillation in a constant volume chamber equipped with an orifice plate
}

\section{PLEASE CITE THE PUBLISHED VERSION}

https://doi.org/10.1080/00102202.2017.1389910

\section{PUBLISHER}

(C) Taylor \& Francis

\section{VERSION}

AM (Accepted Manuscript)

\section{PUBLISHER STATEMENT}

This work is made available according to the conditions of the Creative Commons Attribution-NonCommercialNoDerivatives 4.0 International (CC BY-NC-ND 4.0) licence. Full details of this licence are available at: https://creativecommons.org/licenses/by-nc-nd/4.0/

\section{LICENCE}

CC BY-NC-ND 4.0

\section{REPOSITORY RECORD}

Wei, Haiqiao, Dongzhi Gao, Lei Zhou, Jianfu Zhao, and Rui Chen. 2017. "Experimental Investigation of Turbulent Flame Propagation and Pressure Oscillation in a Constant Volume Chamber Equipped with an Orifice Plate". figshare. https://hdl.handle.net/2134/27468. 


\title{
Experimental Investigation of Turbulent Flame Propagation and Pressure Oscillation in a Constant Volume Chamber Equipped With an Orifice Plate
}

\author{
Haiqiao Wei ${ }^{a}$, Dongzhi Gao ${ }^{a}$, Lei Zhou ${ }^{a}$, Jianfu Zhao ${ }^{a}$, and Rui Chen ${ }^{a, b}$

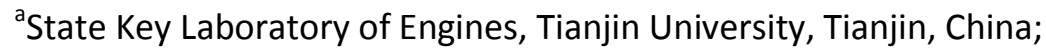 \\ ${ }^{b}$ Department of Aeronautical and Automotive Engineering, Loughborough University, United \\ Kingdom
}

Accepted for publication by Combustion Science and Technology (2017)

\begin{abstract}
In this work, the main contribution is an understanding of different combustion phenomena involving flame acceleration, flame propagation, and the pressure oscillation resulting from flameshock interactions. These physical phenomena were experimentally studied using a newly developed confined combustion chamber equipped with one or two orifice plates. The results showed that there are five stages of flame propagation when a laminar flame passes through the orifice plate in a confined space. These include the deceleration of the laminar flame, jet flame formation and rapid acceleration, deceleration of the flame, turbulent flame formation and acceleration, and turbulent flame propagation in the end-gas region. Flame acceleration and pressure oscillation were found to be strongly related to the aperture size of the orifice plate. The high amplitudes of pressure oscillations were found to be the results of two combustion mechanisms: the end gas auto-ignition and the interactions between the accelerated turbulent flame and shock wave. To further accelerate the flame and promote stronger disturbance in the end gas, another identical orifice plate was employed. Subsequently, strong flame-shock interaction caused end-gas auto-ignition with an extremely high-amplitude pressure oscillation. Eventually, the maximum amplitude of pressure oscillation exceeded $8 \mathrm{MPa}$ as end-gas auto-ignition occurred in the end region of the combustion chamber.
\end{abstract}

KEYWORDS: Auto-ignition; Flame; Orifice plate; Pressure oscillation; Shock wave 


\section{Introduction}

To realize higher thermal efficiency and lower emissions to meet strict laws, engine downsizing with supercharging in spark ignition (SI) gasoline engines has been adopted as a new approach. However, abnormal combustions, such as knock or strong pressure oscillations in downsized SI engines, restrict further improvement in engine efficiency through more advanced levels of downsizing (Misdariis et al., 2015; Pan et al., 2014; Robert et al., 2015). In fact, the mechanism of the knock or super-knock phenomenon in gasoline engines has not been fully understood in the past century. Three theories (Bates et al., 2016; Qi et al., 2015; Robert et al., 2015; Yu and Chen, 2015) exist concerning it: the end-gas auto-ignition theory, the detonation theory, and the flame acceleration theory. Essentially, engine knock is always combined with interactions between the flame and shock wave and a rapid chemical energy release or a process in which some or all of the charge may be consumed at extremely high reaction rates. In other words, the pressure oscillations in a knocking engine are possibly induced by the interaction between the flame and shock wave (Fieweger et al., 1997; Misdariis et al., 2015). Therefore, comprehensively understanding the interaction mechanism between the flame and shock wave is extremely beneficial for avoiding pressure oscillation and improving the engine performance. Thus, in this work we will investigate turbulent flame propagation, flame-shock interactions, and pressure oscillation in a confined combustion chamber. These phenomena can be used to understand the knock mechanism in downsized SI engines.

The present methodologies (Ciccarelli and Dorofeev, 2008; Ciccarelli et al., 2013; Ivanov et al., 2011; Oran and Gamezo, 2007; Petchenko et al., 2007; Pinos and Ciccarelli, 2015; Starr et al., 2015; Zipf et al., 2014) for studying the interaction mechanism between the flame and shock wave usually involve experiments and simulations in long tubes, which can generate the turbulent flame and make flame acceleration to trigger the shock wave and even detonation. For a freely expanding flame, the initially smooth surface of the flame is wrinkled due to Darrieus-Landau instability with thermal diffusion effect. However, in a confined space with obstacles (Ciccarelli and Dorofeev, 2008), the well-known Kelvin-Helmholtz (KH) and Rayleigh-Taylor (RT) instabilities are triggered as the flame passes over an obstacle or through a vent, which could result in the formation of a suddenly accelerated flame. Furthermore, an additional flame surface is produced by the acoustic wave or reflected shock wave due to Richtmyer-Meshkov (R-M) instability (Petchenko et al., 2006). Note that the interaction between the flame and shock wave is always accompanied by the acoustic oscillations, which can lead to significant overpressures and pressure oscillations within a confined space (Thomas et al., 2001; Xiao et al., 2013). However, the amplitude of the pressure oscillations or overpressures is very small. There is still a lack of information on intense pressure oscillation, which is similar to the knock phenomenon in engines.

However, due to the complex and multi-scale instantaneous features, the turbulent flame is a very complex phenomenon and is not easy to control. Researchers are also far from completely understanding the physical process (Bychkov et al., 2008; Chaudhuri et al., 2012; Hof et al., 2005; Landau and Lifshitz, 1987) in the context of the present knowledge system. In addition, turbulent flame propagation is still a vital obstacle for quantitatively understanding and predicting the interaction between the flame and shock (Bychkov et al., 2008). In fact, flame acceleration and propagation are also the most important stages in the interaction between the flame and shock wave for understanding pressure oscillations. A great deal of effort (Ciccarelli and Dorofeev, 2008; Ciccarelli et al., 2010; Dorofeev, 2011;Maley et al., 2015; Oran and Gamezo, 2007; Radulescu and 
Maxwell, 2011; Roy et al., 2004; Xiao et al., 2013) has been spent on studying the turbulent flame acceleration mechanism in channels equipped with and without obstacles in past decades. Recently, Dorofeev (2011) reviewed the basic mechanisms and physical phenomena of flame acceleration in different configurations. Compared to the flame propagation in smooth tubes, a flame in tubes obstructed with obstacles is easier to convert into a turbulent flame. They actually exhibited different flame acceleration mechanisms (Shelkin, 1940). In a sufficiently long smooth tube, a stable flame becomes unstable and forms a turbulent flame over time, and at some stage a shock forms ahead of the flame. The flame acceleration mechanism was strongly controlled by wall roughness (Shelkin, 1940). In contrast, Bychkov et al. (2008) noted that the flame acceleration in long tubes equipped with obstacles was induced by the delayed burning between the obstacles. It can generate a powerful jet flow driving an extremely fast flame velocity (Bychkov et al., 2008; Valiev et al., 2013). Similar to the works by Bychkov et al. (2008), Di Sarli et al. (2012a) used time-resolved particle image velocimetry (TRPIV) and large eddy simulation (LES; Di Sarli et al., 2012b; Di Sarli and Di Benedetto, 2012c, 2013) to investigate the unsteady flame propagation around toroidal vortices generated at the wake of a circular orifice. Their simulation results showed the trend of flame propagation. Previous studies have significantly improved our understanding of the flame acceleration process. Many studies only focused on flame acceleration with obstacles in obstructed channels, but the subsequent turbulent flame self-acceleration and propagation without obstacles due to different instability mechanisms and spatial confinement resulting in pressure oscillation were not completely understood. Therefore, the experimental studies of turbulent flame propagation resulting in shock waves and pressure oscillation in a confined space are still needed to be carried out to understand the knock phenomenon.

The goal of the present study was to further investigate the flame propagation mechanism with the interaction between the flame and shock wave and the pressure oscillation mechanism in a confined space as a preliminary study. The effects of one or two orifice plates with different sizes on flame propagation and pressure oscillation were studied. In the present study, a newly designed experimental apparatus equipped with a multi-orifice plate in a constant volume chamber was employed based on the work by Bychkov et al. (2008) of the flame acceleration mechanism over obstacles. The apparatus may be conveniently used to explore in detail the turbulent flame propagation and the interaction between the shock wave and turbulent flame resulting in pressure oscillation in a confined space. In this study, the orifice plate was different from previous studies (Dorofeev, 2011) on flame propagation in an obstacle channel. Moreover, the orifice plate can generate a very fast accelerated flame more effectively in a small confined space, which can shorten the distance and time from initial flame kernel to fast flame. Laminar flames passing through the orifice plates with different aperture sizes were observed by high-speed Schlieren photography. The present experiment was capable of capturing the evolving combustion-front structure and measuring the flame tip velocity and shock wave velocity. Meanwhile, another object focused on the pressure oscillation mechanism, which was analyzed in detail. In this study, a stoichiometric hydrogen-air mixture was used as the test fuel because of its fast-flame tip velocity, which could be used to investigate the flame-shock interaction. The new experimental apparatus was useful for quantitatively controlling the turbulent flame through the different aperture sizes of the orifice plate. In this work, fundamental studies of the flame-shock interactions were carried out to understand the knock mechanism to avoid strong pressure oscillation in downsized SI engines. 
The article is organized as follows: the experimental setup and conditions are briefly discussed in the next section. In addition, the flame acceleration and pressure oscillation mechanisms are presented in the third section. Finally, major conclusions from this work are drawn in the last section.

\section{Experimental facilities}

This experiment was carried out in a newly designed constant volume combustion bomb equipped with a high-speed Schlieren photography system, as shown in Figure 1. The entire experimental system consisted of a constant volume combustion chamber, a high-speed Schlieren photography system, a pressure recording system, an intake and exhaust system, a high-voltage ignition system, orifice plates with different aperture sizes, a synchronization controller, etc. 


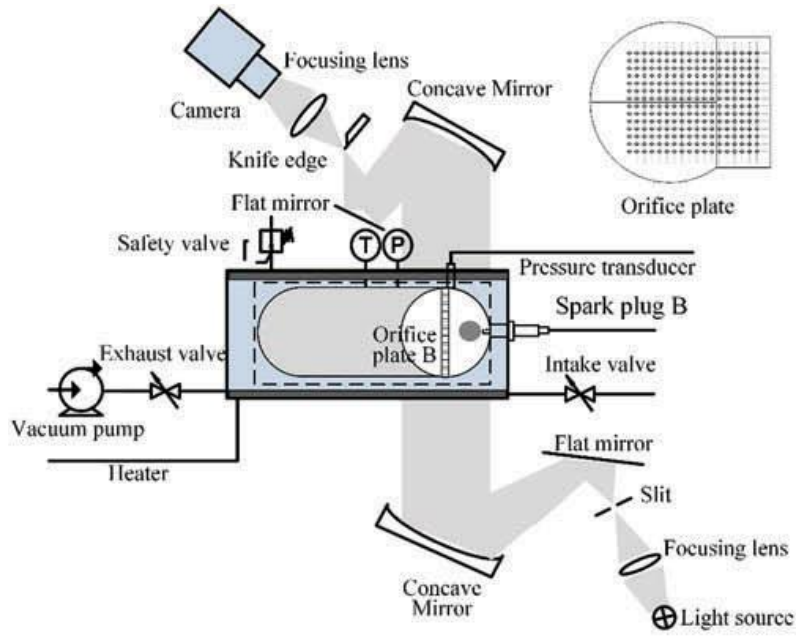

(a)

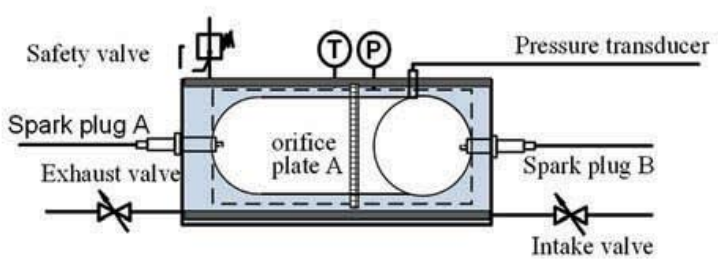

(b)

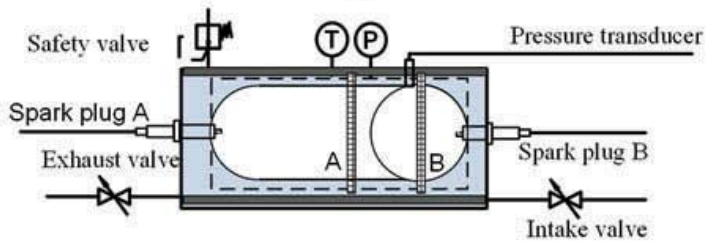

(c)

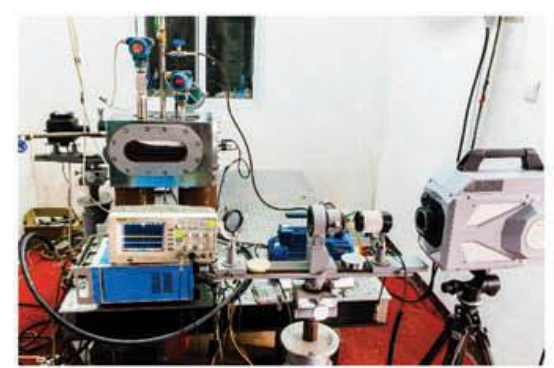

(d)

Figure 1 The schematic diagram of (a) the experimental setup with orifice plate $A$, (b) the experimental setup with orifice plate B, (c) orifice plate, and (d) sketch of optical window.

The combustion chamber was a closed cylindrical cavity with an inner diameter of $100 \mathrm{~mm}$ and length of $230 \mathrm{~mm}$. Replaceable orifice plates with different aperture sizes were installed in the combustion chamber to accelerate the flame and promote the formation of turbulent flame. The orifice plate was constructed using a 3-mm-thick stainless-steel plate with several through-holes in it, which were distributed in a rectangular configuration (18 rows, 14 columns). For safety reasons, an 8-MPa pressure-release valve was installed in the combustion vessel. The entire vessel was uniformly preheated by a set of electrical heating elements with a total power of $2 \mathrm{~kW}$. With the help of a closed-loop feedback controller, the combustion chamber was heated to the target temperature within $2 \mathrm{~K}$. The mixture was ignited using a slightly modified standard ignition plug with 
extended electrodes. The ignition system generated a spark with a duration of $0.7 \mathrm{~ms}$. The combustion images were recorded by the image acquisition system using high-speed Schlieren photography technology at the frame rate of $90,000 \mathrm{fps}$ and exposure time of $1 \mu \mathrm{s}$. The high-speed Schlieren photography technology can capture the density field and was widely used to capture flame and shock wave. The pressure rise during the combustion process was recorded using a Kistler $6113 \mathrm{~B}$ pressure transducer at $100 \mathrm{kHz}$. With the help of the synchronization controller, the spark plug, the pressure recording system, and the high-speed camera started to work at the same time.

In the combustion chamber, there are two positions to mount the orifice plate: position $A$ at the middle of the chamber and position B $28 \mathrm{~mm}$ from the left wall. Moreover, the spark plug can be installed on the left wall or the right wall. For different purposes, there are various methods to arrange the experimental apparatus. As shown in Figure 1a, the spark plug was mounted on the left wall and the orifice plate was mounted at the position B. The flame acceleration, when passing through the orifice plate, could be observed by this method. As shown in Figure $1 \mathrm{~b}$, the orifice plate was mounted at the position $A$, while spark plug $A$ was used to ignite the flammable mixture. Therefore, the combustion phenomenon in the end region of the combustion chamber could be captured. Beyond that, spark plugs $A$ and $B$ were used in an experiment. The spark $A$ was triggered 2.9 ms before spark plug B. Spark plug A was used to induce the main flame and spark plug B was used to mimic the end gas auto ignition. As shown in Figure 1c, the two same orifice plates were used to further accelerate the flame and induce stronger disturbance in the end gas.

In this study, the flame propagation velocity is calculated based on the time derivation of the flame tip position, distance from the ignition point. A velocity point is calculated from the difference in the centerline flame position between consecutive frames. And this was called flame tip velocity in the text. Note that based on a framing rate of 90,000 frames per second and a resolution of 0.18 $\mathrm{mm} /$ pixel, the uncertainty is $16 \mathrm{~m} / \mathrm{s}$.

The experimental conditions are listed in Table 1.Note that the porosities of hole sizes of $1.0 \mathrm{~mm}$, $1.25 \mathrm{~mm}, 1.5 \mathrm{~mm}, 2 \mathrm{~mm}$, and $3 \mathrm{~mm}$ are $1.6 \%, 2.5 \%, 3.6 \%, 6.4 \%$, and $14.4 \%$, respectively. In this study, the uncertainty measures for initial pressure and temperature are no more than 0.05 bar and $2 \mathrm{~K}$, respectively. Each case was carried out three times. Further, in the previous work (Wei et al., 2017) a repeatability test of the experimental setup was shown. Three cases were conducted under the same initial conditions. The tendencies of flame trajectory in three cases were consistent with each other. The relative error did not exceed $5 \%$ and this was acceptable for high-speed turbulent flame propagation after the orifice plate. Thus, the experimental setup for this test is reliable.

\section{Experimental results and discussions}

\section{Flame acceleration}

The flame acceleration process was investigated using the confined chamber equipped with an orifice plate. The orifice plate was located at position B so that the flame propagation could be observed with the high-speed Schlieren photography through the optical window. Several Schlieren images of flame propagation were taken from the experiment with an initial pressure of 4 bar and an aperture size of $1.75 \mathrm{~mm}$ as shown in Figure 2. The flame propagation traveled from right to left. It was found that the entire process of flame propagation after ignition may be divided into two parts: laminar flame propagation and turbulent flame propagation. The fast flame acceleration was 
achieved by the flame passing through the orifice plate, which is similar to the flame acceleration in an obstructed chamber. It should be noted that the fast flame acceleration was accompanied Figure 2. A set of Schlieren images of flames obtained from the test with an orifice plate aperture size of 1.5 $\mathrm{mm}$ (the experimental setup with orifice plate B). by corrugation of the flame shape with fast turbulent burning. From $2.21 \mathrm{~ms}$ to $2.47 \mathrm{~ms}$, it could be seen that the spherical laminar flame was compressed by the orifice plate; consequently, a clearly delayed burning occurred before the flame passed through the orifice plate. Due to the expansion of the burned gas, a flow was formed ahead of the flame. As suggested by Bychkov et al. (2008), the delayed burning between the obstacles created a powerful jet flow, driving the flame acceleration. In Figure 2, the jet flow could be obviously observed from $2.21 \mathrm{~ms}$. When the flame passed through the orifice plate, it was entrained by the powerful flow. Meanwhile, the flame surface was winkled by the orifice and rapidly increased according to the flame instability mechanisms, that is, the well-known KH and RT instabilities. The thermal expansion of the hot combustion products and fast burning rates pushed the flame towards the unburned gas at the right of the orifice plate. After the orifice plate, several jet flames were formed with high speed, as shown from $2.52 \mathrm{~ms}$ to $2.60 \mathrm{~ms}$. Then the jet flames coalesced with each other. Consequently, the fast turbulent combustion was observed in the time interval from $2.67 \mathrm{~ms}$ to $2.90 \mathrm{~ms}$ as shown in Figure $\mathbf{2}$.

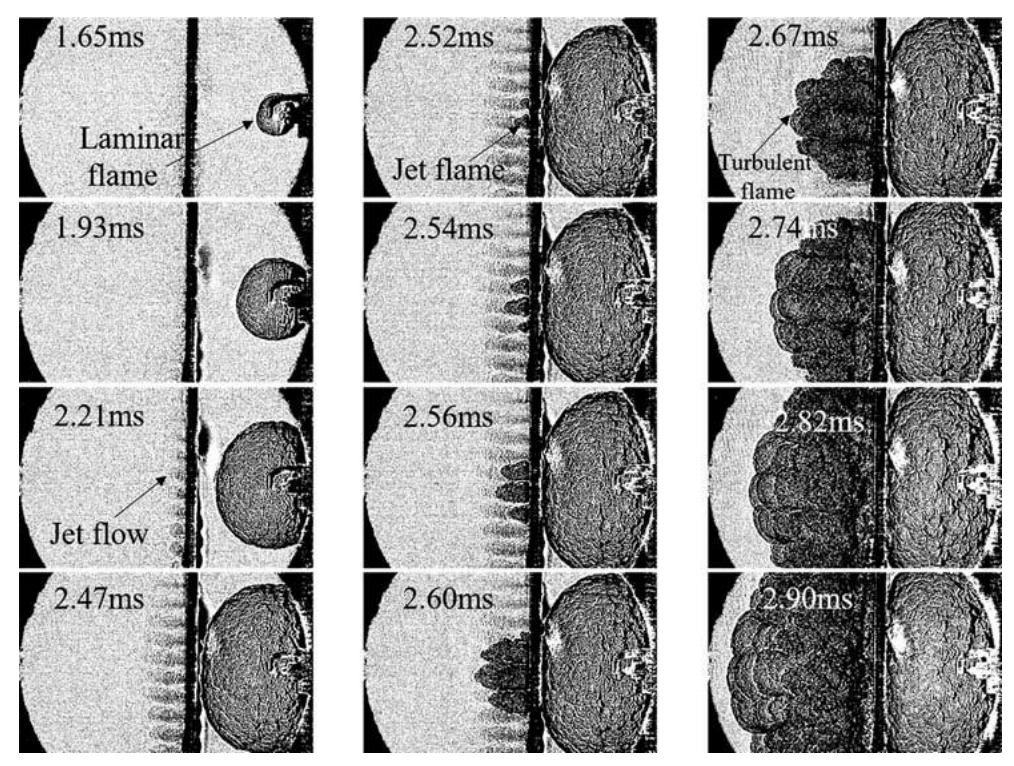

Figure 2 A set of Schlieren images of flames obtained from the test with an orifice plate aperture size of 1.5 mm (the experimental setup with orifice plate B).

Figure 3 qualitatively illustrates the evolution of flame propagation (or flame-tip speed) for different aperture sizes with initial conditions of pressure of 2 bar and stoichiometric hydrogen/air mixture. In Figure 3, the propagation speed of the laminar flame tip was approximately $20 \mathrm{~m} / \mathrm{s}$, which is consistent with the other experiment data by Xiao et al. (2013) under similar conditions. Note that, before the experiment, the ambient gas was quiescence without turbulence and the flame becomes a cellular spherical flame due to the flame instability. Hence, the spherical flame was defined as the laminar flame. It was found that the laminar flame tip velocity decreased gradually before passing through the orifice plate, as shown in Figure $3 \mathrm{~b}$. The increase of the aperture size of the orifice plate made the flame less confined and the decreasing trend of the laminar flame became slow. The expanding flame was formed once the laminar flame passed through the orifice plate. A rapid increase in flame tip velocity was observed. The flame flowing across the orifice resulted in a rapid 
increase of the size of the turbulent flame surface. In addition, the flame propagation changed direction to the axial part of the orifice, which accumulated into a strong jet and consequently drove the flame-front rapidly forward. Meanwhile, KH-RT instability also led to an additional increase in flame surface area. As indicated in Bychkov et al. (2008), burned gas flowed out of the orifice plate with the speed $\left|u_{x}\right|=(\Theta-1) U_{f}$, where $U_{f}$ is the laminar speed and $\Theta$ is the expansion factor, which is typically $\Theta=5-8 \Theta$. In this work, the mean velocity of the flame front flowing out of the orifice plate was consistent with this equation as shown in Figure 3. After the acceleration, the flame-front tip velocity decreased because of a fluid dynamic mechanism that the velocity of a fluid passing through a small space (orifice plate) with a relatively larger pressure to a large space with relatively smaller pressure will decrease. At the post-flame propagation stage, the turbulent flame was formed and the flame self-acceleration continued to drive the flame-front tip velocity increase. Here, the post-flame propagation stage means the obvious jet flame disappeared and onset of turbulent flame formation occurred, as shown in Figure 3a. It was noted that in Bychkov theory the ultrafast flame acceleration in obstructed channels is due to laminar flame acceleration. However, in this work the jet flame was formed after the laminar flame passed through the orifice plate and subsequently the turbulent flame was generated. In previous works (Bradley et al., 2000; Wu et al., 2013), the flame self-acceleration had been validated. Note that in a confined space the pressure could increase following the turbulent combustion process (Shy et al., 2015), which would further increase the flame tip velocity. In summary, the overall trend of flame acceleration does not change.

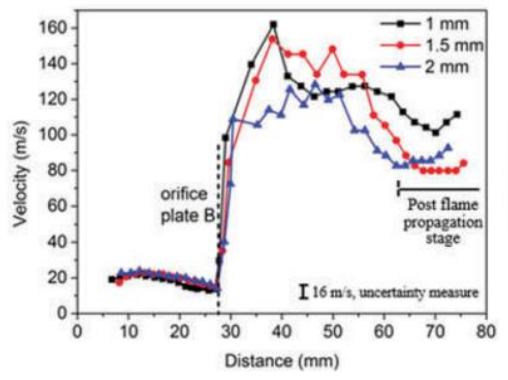

(a)

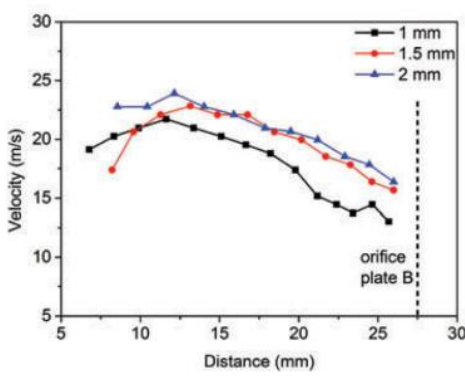

(b)

Figure 3 Evolution of flame front tip velocity for different aperture sizes at initial pressure of $\mathbf{2}$ bar.

The flame passing through the orifice plate with a small aperture size leads to a large increase in the flame surface area and small eddy structures. In other words, the effect of a small aperture size of orifice plate is equivalent to the perturbation of many small eddies on the flame front, which consequently leads to a strong turbulent flame. Thus, a monotonic relationship between the turbulent flame-front tip velocity and the aperture size of the orifice plate is shown in Figure 4. Note that, in this work, the change of aperture size will lead to the change of porosity in the present experiment, which also has an effect on the final flame velocity. The mean turbulent flame-front tip velocity only represents the flame-front tip velocity passing through the orifice plate to the end of the optical window. In this work, the orifice plate with the present aperture sizes influences flame acceleration positively. 


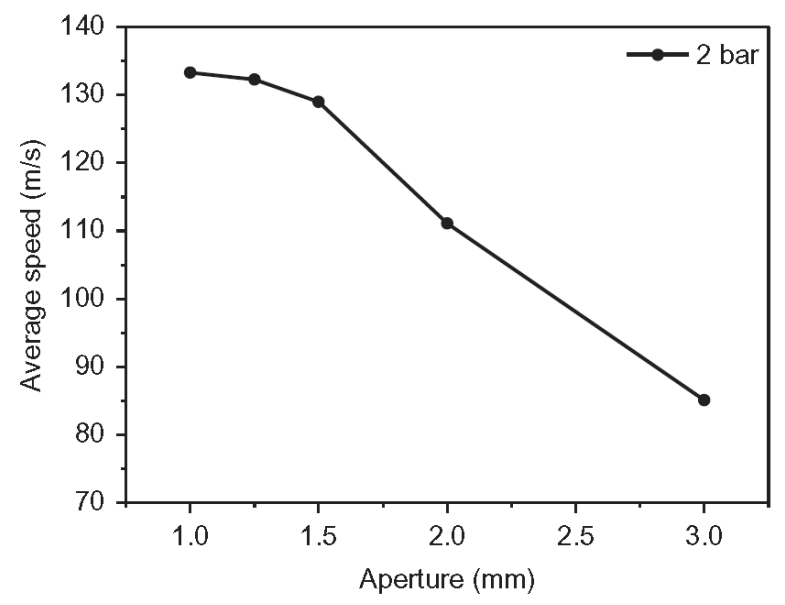

Figure 4 Average turbulent flame-front tip velocity induced by different aperture sizes of $1 \mathrm{~mm}, 1.25 \mathrm{~mm}, 1.5 \mathrm{~mm}, 2 \mathrm{~mm}$, and $\mathbf{3} \mathrm{mm}$ at initial pressure of $\mathbf{2}$ bar.

\section{Flame propagation and pressure oscillation}

To clearly observe the interactions between turbulent propagation and shock waves and investigate the pressure oscillation mechanism, the orifice plate (plate A) placed at the middle position of the chamber was used. The flame propagation proceeded from left to right in Figure 5. A set of Schlieren images describing the flame propagation process and shock-flame interaction were clearly shown in Figure 5 at the initial pressure of 4 bar and aperture size of $1.5 \mathrm{~mm}$. A clear density disturbance ahead of the turbulent flame (or preheated zone with high density) in the unburned gas zone was formed. Subsequently, a shock wave (Mach number about unity) was initially observed due to the turbulent flame acceleration. After that, a shock wave passed through the disturbed unburned gas and consequently a preheated zone was formed between the shock wave and the flame front, as seen at $4.139 \mathrm{~ms}$. From images at $4.22 \mathrm{~ms}, 4.231 \mathrm{~ms}$, and $4.255 \mathrm{~ms}$, it was found that the turbulent flame continues to accelerate and further intensify the combustion. In fact, the preheated zone is important for forming strong turbulent combustion and autoignition of unburned mixture ahead of the turbulent flame. Although it is difficult from the current images to distinguish whether the combustion phenomena is the denotation or not, the present results, including the obvious preheated zone between the flame front and leading shock wave, are similar to the formation process of deflagration-to-detonation transition shown in previous research (Liberman et al., 2010).
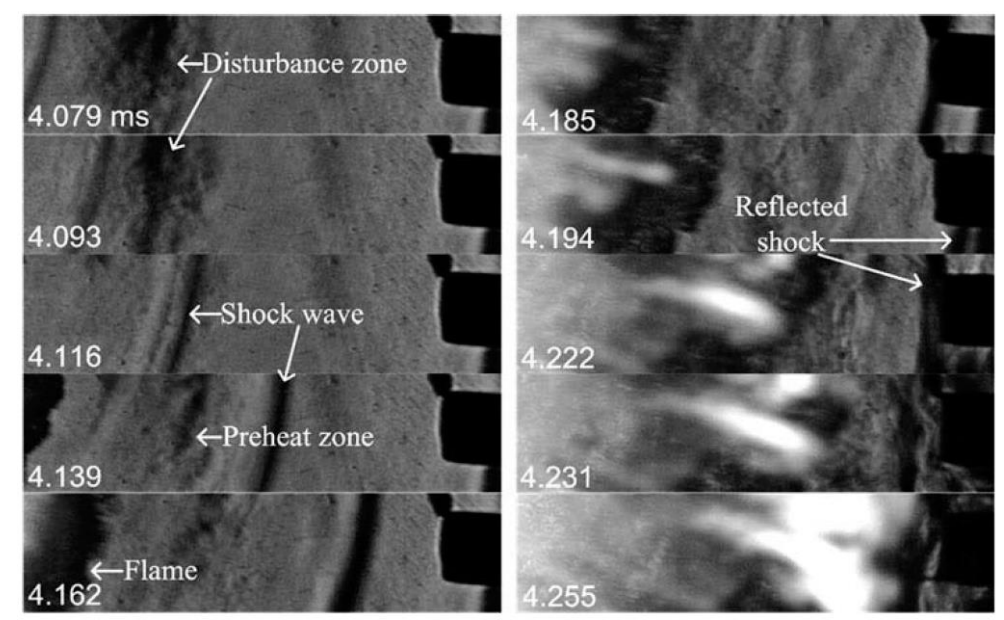

Figure 5 Series of Schlieren images of flame and shock wave propagation. 
Note that the reflected shock also contributes to the turbulent combustion, and the flame-shock interaction induces the strong pressure oscillation, as shown in detail in Figure 6 . The pressure oscillation was strongly related to the different turbulent combustion stages in the confined chamber. The first peak of pressure oscillation occurred when the shock wave passed through the pressure transducer at approximately $4.185 \mathrm{~ms}$. In addition, the largest amplitude of pressure oscillation generated by the interaction between the turbulent flame and reflected shock wave was observed at approximately $4.3 \mathrm{~ms}$. The pressure oscillation is similar to the knock in SI engines. Although there is a large pressure oscillation under the conditions of initial pressure of 4 bar and aperture size of $1.5 \mathrm{~mm}$, the end-gas auto ignition has not been visualized clearly. Moreover, a strong pressure oscillation is generated because of the interactions between the flame and the shock wave, resulting in auto-ignition between them. In addition, Figure 6 shows that the small aperture size of the orifice plate induced to a higher amplitude of pressure oscillation than the large aperture size of the orifice plate. The reason can be explained from Figure 3. In comparison, pressure oscillation in the case without the orifice plate was very weak due to a low flame tip velocity, which will be discussed later. Note that increasing the turbulent flame intensity by reducing the aperture size of the orifice plate can also increase the burning rate and release more energy driving the pressure to rise rapidly.

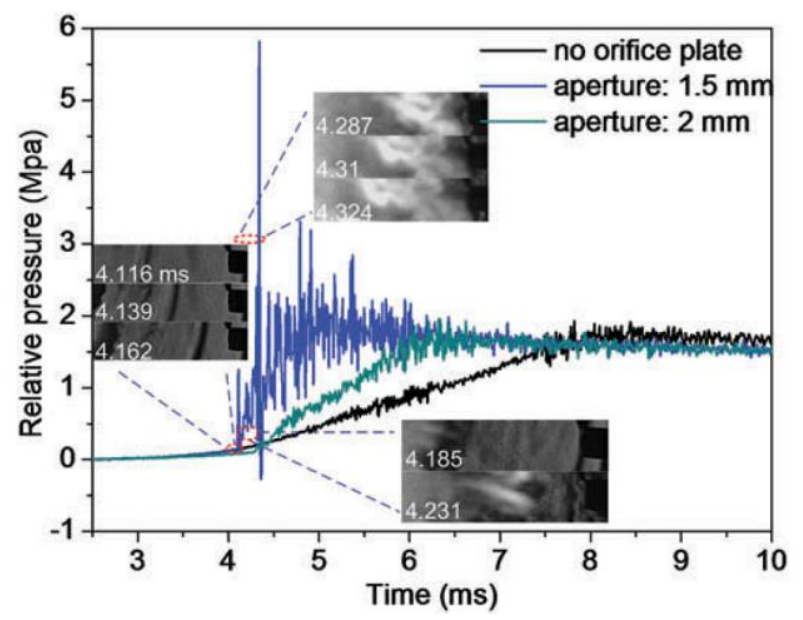

Figure 6 Pressure oscillations at two aperture sizes of $1.5 \mathrm{~mm}$ and $2 \mathrm{~mm}$ compared with the case without orifice plate.

Figure 7 shows the comparisons of flame front velocities and reflected shock wave velocity versus distance. The flame propagation velocity is calculated from the time derivative of the flame tip location. It is worth noting that the right edge of the images is the end wall of the combustion chamber. It can be seen in Figure $\mathbf{7}$ that the flame-front tip velocity for the case without an orifice plate demonstrates a decreasing trend due to the influence of gas compression in the end-gas region of a confined space, which is a well-known feature. For the case with the aperture size of $2 \mathrm{~mm}$, it can be seen that the flame front tip velocity also decelerated partly due to confined space limitations, which is similar to laminar flame propagation in a confined space. Furthermore, the interaction of the flame front with the reflected pressure wave or acoustic wave from the wall also decelerated the flame-front tip velocity and generated an oscillating combustion phenomenon. It was found that the interaction between the flame front and the shock wave with a velocity of approximately $470 \mathrm{~m} / \mathrm{s}$ led to the flame acceleration, successively triggered by the emergence of autoignition between the flame and the shock wave, which clearly enhanced the combustion. In terms of the results in Figures 6 and 7, the strong flame tip velocity could cause a high energy 
release rate to generate a strong compression effect and shock wave. As the autoignition occurred, that could result in a high amplitude of pressure oscillation. Overall, the pressure oscillation is related to the turbulent flame velocity.

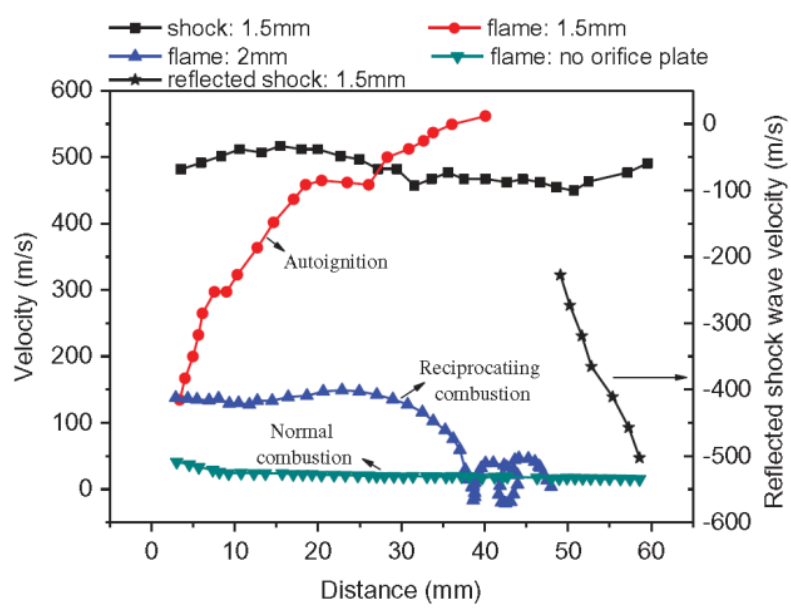

Figure 7 The trace histories of flame speed and shock wave with and without orifice plate at different positions.

Based on the above studies, the evolution mechanism of flame propagation in a confined combustion chamber was summarized in the sketch in Figure 8. It can be clearly shown that there are five stages for the turbulent flame formation and propagation when the laminar flame passes through the orifice plate in the confined space; that is, the deceleration of the laminar flame, jet flame formation and rapid acceleration, deceleration of the turbulent flame, subsequent acceleration of the turbulent flame, and turbulent flame propagation in the end-gas region. Moreover, at the fifth stage of flame propagation in the end-gas region, the turbulent flame has fully developed and its development is influenced by the confined space and acoustic wave or reflected shock wave. It was found that the flame acceleration in the end-gas region occurs only when the auto ignition of unburned gas between the flame and shock wave occurs, as shown in Figure 7. The reciprocating combustion was formed because of the backward movement of the flame induced by the compression of the reflected shock wave or acoustic wave. In theory, when the acoustic wave becomes sufficiently weak, normal combustion occurs, causing the flame tip velocity to slowly decrease, and the flame moves forward constantly until it reaches the wall. It is worth noting that at the fourth stage of the flame propagation, the flame self-acceleration is not fully observed in the present work. However, the flame acceleration process should be divided into two processes: turbulent flame self-acceleration and flame acceleration due to the R-M instability mechanism. However, the fourth stage of turbulent flame propagation occurs beyond the range of our experimental optical window; observation of this stage is our next work. 


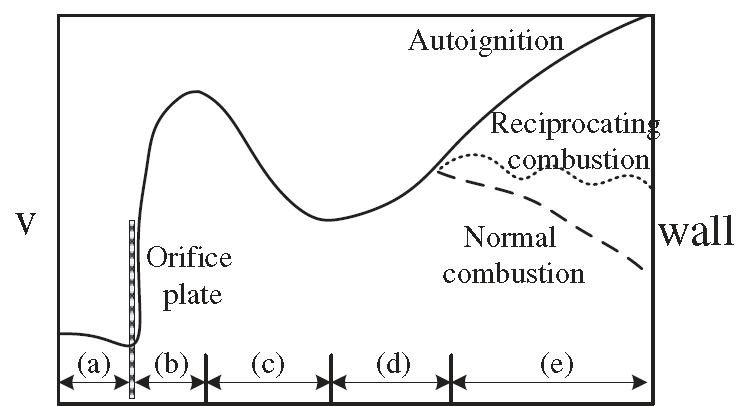

(a)Laminar flame deceleration; (b)Jet flame formation and rapid acceleration; (c)Flame speed deceleration; (d)Turbulent flame acceleration; (e)Flame propagation in the end gas

Figure 8 Sketch of different stages of flame propagation in a confined space equipped with the orifice plate.

\section{Strong pressure oscillation with the end-gas auto ignition}

To further observe the pressure oscillation induced by the interaction between the turbulent flame and shock wave in the end-gas region, in this section, the second orifice plate (plate B) with the same aperture size as plate $A$ located at the optical window in the end-gas region was added. The orifice plate $B$ together with the first orifice plate $A$ was employed to further accelerate the turbulent flame. Meanwhile, a (second) spark plug mounted in the end-gas region was used to ignite the hydrogen-air mixture at the end-gas region, as shown in Figure 1, which was intended to mimic the hot spot. However, according to present results, the spark ignition was a little different from the hot spot auto-ignition process under the present experimental conditions, which cannot lead to deflagration or detonation combustion and pressure oscillation. This is because the single hot spot cannot lead to a fast heat release and pressure rise and the auto-ignition is related to a gradient of the reactivity mechanism, according to Zeldovich's theory (Zeldovich, 1980). Meanwhile, Robert et al. (2015) also determined that the auto ignition spots coupled with a pressure wave can result in a detonation, according to LES in an SI engine. Therefore, the spark ignition in the end-gas region can also be used to clearly observe the effect of a shock wave on the laminar flame surface, as shown in Figure 9. The time interval between the first spark ignition and the second spark ignition is $2.9 \mathrm{~ms}$, and the second spark ignition is located at the end of the optical window, as shown in Figure 9. Figure 9 shows the interactions of the shock wave with the turbulent flame with a velocity of approximately $350 \mathrm{~m} / \mathrm{s}$ and the laminar flame with an average velocity of $20 \mathrm{~m} / \mathrm{s}$. It can be seen that the shock wave significantly impacts the laminar flame. From $4.51 \mathrm{~ms}$ to $4.63 \mathrm{~ms}$ the laminar flame was compressed by the shock wave and forced to move backward. However, for the turbulent flame, there were no obvious effects and the turbulent flame sustained forward movement until touching the laminar flame due to the faster turbulent flame compensating for the influence of the shock wave. Consequently, intense gas compression was produced in the end-gas region near the wall. Figure 9 also explains the normal combustion at the fifth stage of flame propagation in the end-gas region, as shown in Figure 8.

Figure $9 \mathrm{~b}$ illustrates that the turbulent flame (left), with a velocity of approximately $250 \mathrm{~m} / \mathrm{s}$, and the laminar flame (right), with an average velocity of $20 \mathrm{~m} / \mathrm{s}$, were influenced by the shock wave and reflected shock wave, respectively. A very strong compression zone with high density to the right of the second orifice plate $B$ was formed gradually while the turbulent flame passed through the second orifice plate B at $4.89 \mathrm{~ms}$. In addition, a fast expanding flame in the compression zone 
occurred, which also has a strong compression effect on the laminar flame as clearly seen at $4.96 \mathrm{~ms}$, and flattened the laminar flame. Consequently, the fast flame region led to an additional compression effect on the end gas near the wall due to the thermal expansion and finally triggered the end-gas auto ignition. The intense combustion occurred with a velocity of approximately 700 $\mathrm{m} / \mathrm{s}$ as a high luminous region appeared at $5.04 \mathrm{~ms}$, due to the end-gas auto ignition near the wall as shown in Figure 9. Note that the high luminous region represents the high-intensity combustion. The auto-ignition flame swept through the whole test window in the image at $5.13 \mathrm{~ms}$, which presented a higher flame luminous intensity. It can be concluded that the mechanism of end-gas auto ignition, which results in strong pressure oscillation in a confined space, was formed by the shock wave and the strong compression effect on the end-gas with high density. The compression effect further boosted the energy concentration in the end-gas region. Note that, in the present experiment, an intense combustion zone was formed before the turbulent flame passed through the orifice plate. More detailed information can be observed in Videos S1 and S2 in the supplementary materials.
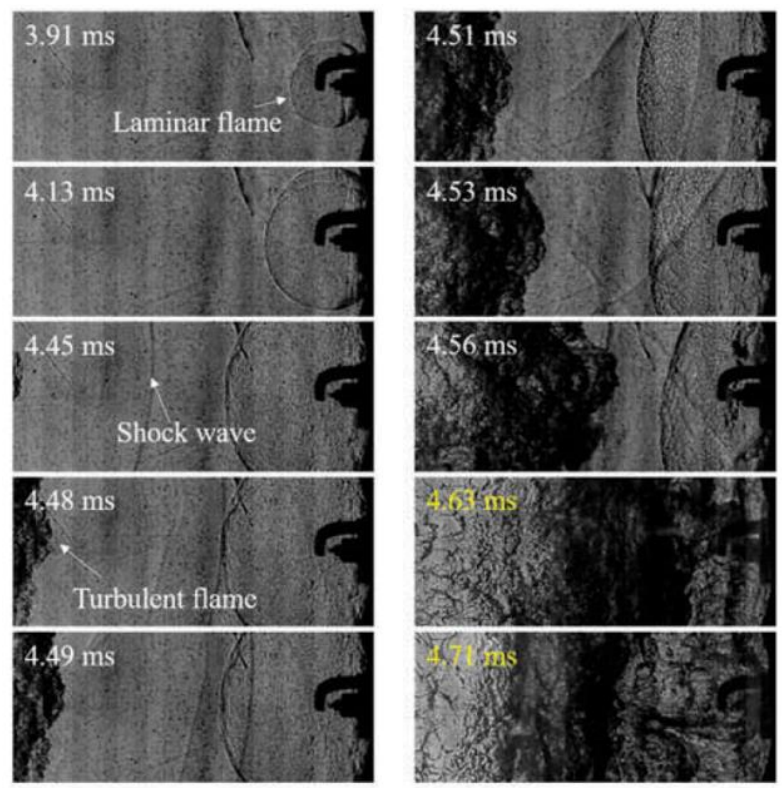

a) With one orifice plate $\mathrm{A}$

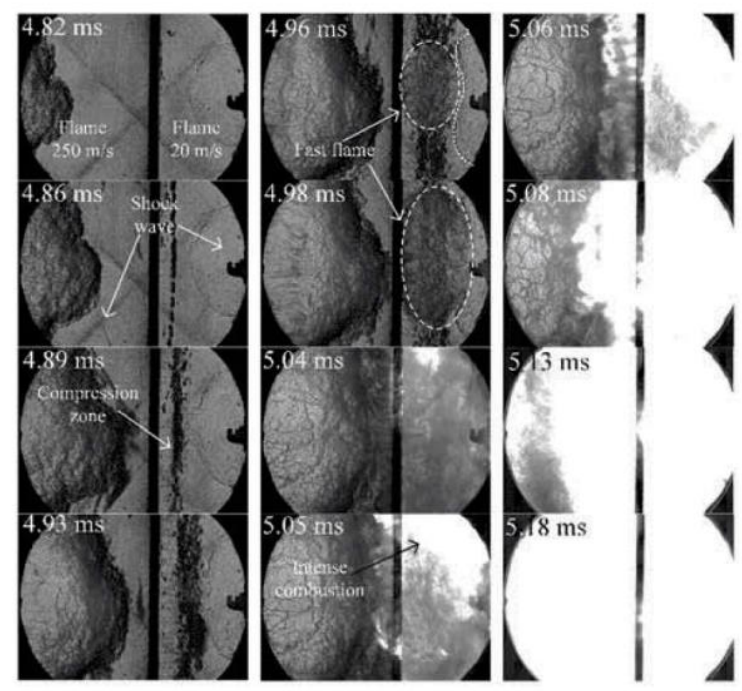

b) With two orifice plates $\mathrm{A}$ and $\mathrm{B}$ 
A strong pressure oscillation induced by the violent combustion is shown in Figure 10. It was slightly different from that shown in Figure 6 , in which the auto ignition occurred between the shock wave and turbulent flame. The maximum amplitude of the pressure oscillation, which was more than 8 $\mathrm{MPa}$, was obtained due to end-gas auto ignition. In comparison, the same setup except without the second orifice plate was employed as a comparison test. Note that, for the test without the orifice plate, only the phenomenon of the shock wave compressing the turbulent flame and laminar flame was observed. Thus, the amplitude of the pressure oscillation was small due to the absence of endgas auto ignition. The present results also indicate that the strong compression effect on the end-gas could lead to violent combustion and strong pressure oscillations, which have a destructive influence on the device. The strong combustion wave causes an extreme enough force to bend the orifice plate, which can be seen in Video $\$ 2$ in the supplementary materials.

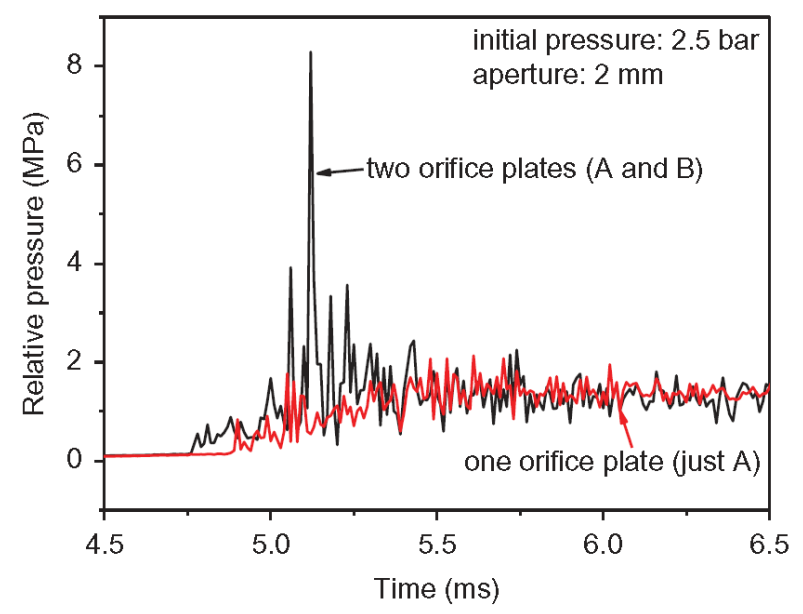

Figure 10 Pressure oscillations with two orifice plates compared with the case with one orifice plate A.

\section{Conclusions}

In this work, a newly designed apparatus was used to investigate the turbulent flame propagation and pressure oscillation process with a stoichiometric hydrogen-air mixture. The turbulent flame formation and propagation, shock-flame interactions, and the end-gas auto ignition were visualized using high-speed Schlieren photography in a confined chamber equipped with an orifice plate. The results showed that there are five stages for the turbulent flame formation and propagation when a laminar flame passes through an orifice plate in a confined space, that is: (1) the deceleration of the laminar flame, (2) jet flame formation and suddenly rapid acceleration, (3) deceleration of the flame, (4) the acceleration of the turbulent flame, and (5) turbulent flame propagation in the end-gas region. A delayed burning between the laminar flame and the orifice plate was observed clearly in the present work, which experimentally demonstrates the Bychkov work. For flame acceleration over the orifice plate, a central jet flow was formed and subsequently a rather strong jet flame with the corrugated flame shape and fast turbulent burning was generated, which is consistent with the previous theory's analysis. It should be noted that, at the fifth stage of flame propagation in the endgas region, there were three possible features of flame propagation, including flame sustained acceleration, flame reciprocating motion, and flame deceleration. Moreover, an approximately linear relationship of turbulent flame front tip velocity with the aperture size of the orifice plate was observed under the present experimental conditions. The orifice plate with small aperture size was capable of producing a high-amplitude pressure oscillation in this work. 
It was clear from the present study that the turbulent flame continuously accelerated and further intensified the combustion. The reflected shock also contributed to the strong turbulent combustion and consequently led to the strong pressure oscillation. Moreover, an extremely high amplitude of pressure oscillation phenomenon resulting from the end-gas auto ignition was observed when the second orifice plate was also used to form a strong compression zone. The maximum amplitude of the pressure oscillation was capable of more than $8 \mathrm{MPa}$, which was of huge destructive capacity to the experimental device. It may be concluded that the mechanism of the end-gas auto ignition, which results in strong pressure oscillation in a confined space, consists of the shock wave's influence on the temperature and density in the end-gas region and the strong compression effect on the end gas with a very high density. Overall, the present results provided a new insight into the flame acceleration and propagation and pressure oscillation formation mechanism in confined space, allowing the capability to better understand the knock and super-knock mechanisms in SI engines.

\section{Funding}

This work was supported by the National Natural Science Foundation of China (Grant Nos. 51476114, 91641203, and 51606133).

\section{References}

Bates, L., Bradley, D., Paczko, G., and Peters, N. 2016. Engine hot spots: Modes of auto-ignition and reaction propagation. Combust. Flame, 166, 80-85.

Bradley, D., Sheppart, C., Woolley, R., Greenhalgh, D., and Lockett, R. 2000. The development and structure of flame instabilities and cellularity at low Markstein numbers in explosions. Combust. Flame, 122, 195-209.

Bychkov, V., Valiev, D., and Eriksson, L.-E. 2008. Physical mechanism of ultrafast flame acceleration. Phys. Rev. Lett., 101, 164501.

Chaudhuri, S., Wu, F., Zhu, D., and Law, C.K. 2012. Flame speed and self-similar propagation of expanding turbulent premixed flames. Phys. Rev. Lett., 108, 044503.

Ciccarelli, G., and Dorofeev, S. 2008. Flame acceleration and transition to detonation in ducts. Prog. Energy Combust., 34, 499-550.

Ciccarelli, G., Johansen, C., and Kellenberger, M. 2013. High-speed flames and DDT in very roughwalled channels. Combust. Flame, 160, 204-211.

Ciccarelli, G., Johansen, C.T., and Parravani, M. 2010. The role of shock-flame interactions on flame acceleration in an obstacle laden channel. Combust. Flame, 157, 2125-2136.

Di Sarli, V., and Di Benedetto, A. 2012c. Sensitivity to the presence of the combustion submodel for large eddy simulation of transient premixed flame-vortex interactions. Ind. Eng. Chem. Res., 51, 7704-7712.

Di Sarli, V., and Di Benedetto, A. 2013. Effects of non-equidiffusion on unsteady propagation of hydrogen-enriched methane/air premixed flames. Int. J. Hydrogen Energy, 38, 7510-7518. 
Di Sarli, V., Di Benedetto, A., Long, E.J., and Hargrave, G.K. 2012a. Time-resolved particle image velocimetry of dynamic interactions between hydrogen-enriched methane/air premixed flames and toroidal vortex structures. Int. J. Hydrogen Energy, 37, 16201-16213.

Di Sarli, V., Di Benedetto, A., and Russo, G. 2012b. Large eddy simulation of transient premixed flame-vortex interactions in gas explosions. Chem. Eng. Sci., 71, 539-551.

Dorofeev, S.B. 2011. Flame acceleration and explosion safety applications. Proc. Combust. Inst., 33, 2161-2175.

Fieweger, K., Blumenthal, R., and Adomeit, G. 1997. Self-ignition of SI engine model fuels: A shock tube investigation at high pressure. Combust. Flame, 109, 599-619.

Hof, B., van Doorne, C.W., Westerweel, J., and Nieuwstadt, F.T. 2005. Turbulence regeneration in pipe flow at moderate Reynolds numbers. Phys. Rev. Lett., 95, 214502.

Ivanov, M., Kiverin, A., and Liberman, M. 2011. Hydrogen-oxygen flame acceleration and transition to detonation in channels with no-slip walls for a detailed chemical reaction model. Phys. Rev. E, 83, 056313.

Landau, L.D., and Lifshitz, E.M. 1987. Fluid Mechanics, Elsevier, England.

Liberman, M., Ivanov, M., Kiverin, A., Kuznetsov, M., Chukalovsky, A., and Rakhimova, T. 2010. Deflagration-to-detonation transition in highly reactive combustible mixtures. Acta Astronaut., 67, 688-701.

Maley, L., Bhattacharjee, R., Lau-Chapdelaine, S.-M., and Radulescu, M.I. 2015. Influence of hydrodynamic instabilities on the propagation mechanism of fast flames. Proc. Combust. Inst., 35, 2117-2126.

Misdariis, A., Vermorel, O., and Poinsot, T. 2015. LES of knocking in engines using dual heat transfer and two-step reduced schemes. Combust. Flame, 162, 4304-4312.

Oran, E.S., and Gamezo, V.N. 2007. Origins of the deflagration-to-detonation transition in gasphase combustion. Combust. Flame, 148, 4-47.

Pan, J., Shu, G., and Wei, H. 2014. Interaction of flame propagation and pressure waves during knocking combustion in spark-ignition engines. Combust. Sci. Technol., 186, 192-209.

Petchenko, A., Bychkov, V., Akkerman, V.Y., and Eriksson, L.-E. 2006. Violent folding of a flame front in a flame-acoustic resonance. Phys. Rev. Lett., 97, 164501.

Petchenko, A., Bychkov, V., Akkerman, V.Y., and Eriksson, L.-E. 2007. Flame-sound interaction in tubes with nonslip walls. Combust. Flame, 149, 418-434.

Pinos, T., and Ciccarelli, G. 2015. Combustion wave propagation through a bank of cross-flow cylinders. Combust. Flame, 162, 3254-3262.

Qi, Y., Wang, Z., Wang, J., and He, X. 2015. Effects of thermodynamic conditions on the end gas combustion mode associated with engine knock. Combust. Flame, 162, 4119-4128. 
Radulescu, M.I., and Maxwell, B.M. 2011. The mechanism of detonation attenuation by a porous medium and its subsequent re-initiation. J. Fluid Mech., 667, 96-134.

Robert, A., Richard, S., Colin, O., and Poinsot, T. 2015. LES study of deflagration to detonation mechanisms in a downsized spark ignition engine. Combust. Flame, 162, 2788-2807.

Roy, G., Frolov, S., Borisov, A., and Netzer, D. 2004. Pulse detonation propulsion: Challenges, current status, and future perspective. Prog. Energy Combust., 30, 545-672.

Shelkin, K. 1940. Influence of tube walls on detonation ignition. J. Exp. Theor. Phys., 10, 823.

Shy, S.S., Liu, C.C., Lin, J.Y., Chen, L.L., Lipatnikov, A.N., and Yang, S.I. 2015. Correlations of highpressure lean methane and syngas turbulent burning velocities: Effects of turbulent Reynolds, Damköhler, and Karlovitz numbers. Proc. Combust. Inst., 35, 1509-1516.

Starr, A., Lee, J.H., and Ng, H.D. 2015. Detonation limits in rough walled tubes. Proc. Combust. Inst., 35, 1989-1996.

Thomas, G., Bambrey, R., and Brown, C. 2001. Experimental observations of flame acceleration and transition to detonation following shock-flame interaction. Combust. Theor. Model., 5, 573-594.

Valiev, D.M., Akkerman, V.Y., Kuznetsov, M., Eriksson, L.-E., Law, C.K., and Bychkov, V. 2013. Influence of gas compression on flame acceleration in the early stage of burning in tubes. Combust. Flame, 160, 97-111.

Wei, H., Gao, D., Zhou, L., Feng, D., and Chen, R. 2017. Different combustion modes caused by flameshock interactions in a confined chamber with a perforated plate. Combust. Flame, 178, 277-285.

Wu, F., Jomaas, G., and Law, C.K. 2013. An experimental investigation on self-acceleration of cellular spherical flames. Proc. Combust. Inst., 34, 937-945.

Xiao, H., Shen, X., Guo, S., and Sun, J. 2013. An experimental study of distorted tulip flame formation in a closed duct. Combust. Flame, 160, 1725-1728.

Yu, H., and Chen, Z. 2015. End-gas autoignition and detonation development in a closed chamber. Combust. Flame, 162, 4102-4111.

Zeldovich, Y.B. 1980. Regime classification of an exothermic reaction with nonuniform initial conditions. Combust. Flame, 39, 211-214.

Zipf, R.K., Gamezo, V.N., Mohamed, K.M., Oran, E.S., and Kessler, D.A. 2014. Deflagration-todetonation transition in natural gas-air mixtures. Combust. Flame, 161, 2165-2176. 